\title{
Non -invasive new techniques in oculoplastic
}

\author{
Tsioumas G Sotiris ${ }^{1 *}$, Georgiadis Nikolaos ${ }^{2}$ and Georgiadou Irini $^{3}$ \\ ${ }^{1}$ Private Clinic, Diagnostic Clinic and Aesthetic Surgery Ophthalmology, Eye surgeon - Oculoplastic, President of Hellenic Society of Aesthetic Medicine and \\ Non-Invasive Surgery, Greece \\ ${ }^{2}$ Private Clinic, Professor of Ophthalmology, Aristotle University of Thessaloniki, Greece \\ ${ }^{3}$ Private Clinic, Ph.D. of Aristotle University of Thessaloniki, Greece
}

\begin{abstract} scars, syringomi, papillomi, benign tumors, nodules and chalazion in difficult areas such as gray line. after 2-3 sessions. without complications in his clinic.

\section{Introduction}

In 2017, due to the fact that the advancements in the medical industry are changing rapidly, the need for direct and non-painful solutions to patient's problems is increasing. The Plasma Exeresis method covers that need because the use of it is getting necessary in Oculoplastic and in the Ophthalmology field. Now the doctor is able to propose outright solutions to the patient without mentioning the word surgery, thus eliminate the fear of the patient for the surgery. Our aim is to show the new bloodless techniques in oculoplastic which effectiveness equals that of surgery but without the complications and discomfort that may occur from a surgery.
\end{abstract}

Purpose: The objective of the research is to highlight the new non - invasive techniques there are in oculoplastic aiming in resolving multiple eyesores problems in the ophthalmic area. We will talk about how through new techniques in oculoplastic we can solve problems like, xanathelasma, hemangiomas, cysts, ectropion, entropion,

Method: The methodology that is followed to solve the above-mentioned cases is the Plasma Exeresis using PLEXR machine. After first diagnosis and analysis in each region, we proceed to resolve them using the Plasma Exeresis. The techniques of Plasma Exeresis are either spot by spot or spray method, depending on the case. There is no cauterization because the area is not cauterized but sublimated without complications, scarring, and effect of the optic nerve.

Results: Complete resolution of problem areas from the first session without problems of recurrence. In case such as ectropion and entropion resolution may occur

Conclusion: In the past the solution to such problems required surgical rooms, microscopes, stitches, and long period of recuperation. Today, not only the patient is able to get rid of these specific problems, bloodlessly more economically, without surgery and rehabilitation but also the doctor is able to perform all these methods,

\section{Diagnosis}

We examined and studied 347cases and 147 patients who were experiencing problems such as xanthelasmata (38), hemangiomas (4), cysts (20), entropion (2) ectropion (10), syringomi (100), papillomi (100) benign tumors (45), chalazion (8) and nodules in difficult areas such as the gray line (20). The age range is from $25-78$ years of age, smoking and non- smoking men and women.

All incidents had in common the fear of surgery which explains why the suggestive method was Plasma Exeresis.

\section{Method}

All patients accepted antisepsis in the areas that we would apply the Plasma Exeresis method. Then in all cases, except from those of ectropion, entropion, chalazion and tumors in the gray line, we applied numbing cream for 30 minutes. After the time elapsed we removed the cream and started the process. The area was anesthetized by applying circular anesthesia with the white pistol and the patient did not feel anything during the treatment. In cases of ectropion, entropion, chalazion and tumors in the gray line, we applied numbing injection with xylocaine.

Once anesthetized the area either with anesthetic cream or anesthetic injection we started implementing Plasma Exeresis. We used Plexr machine which has three pistols with different intensity. Depending on the area and the problem, we use the related pistol. Sublimation methods are 2. Spot by spot method or Spray Method. All cases were treated with the two methods either separately or in combination.

\section{Analysis}

Xanthelasma: Spray Method (Figure 1)

Hemangiomas: Spot by spot method (Figure 2)

Cysts: depending on the shape and size of the cyst, combination of 2 methods (Figure 3)

Entropion - Ectropion : Spot by spot with specific direction Scars: Combination of 2 techniques depending on the scar

Correspondence to: Tsioumas G. Sotiris, Private Clinic, Diagnostic Clinic and Aesthetic Surgery Ophthalmology, Eye surgeon - Oculoplastic, President of Hellenic Society of Aesthetic Medicine and Non-Invasive Surgery, Greece.

Received: September 30, 2017; Accepted: October 18, 2017; Published: October 21,2017 


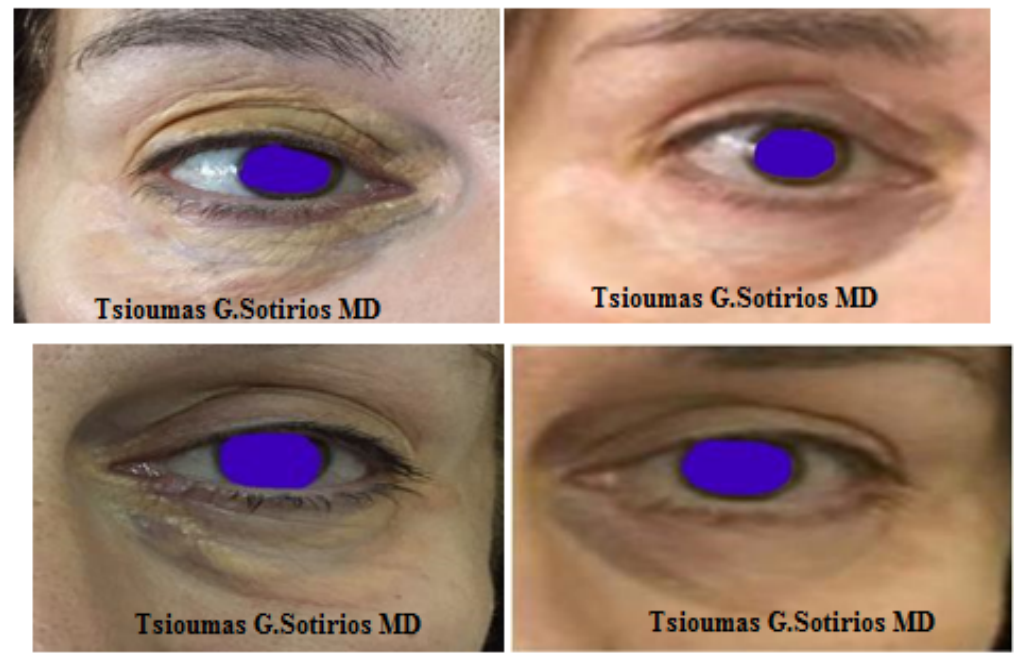

Figure 1. Removal of xanthelasma with plexr

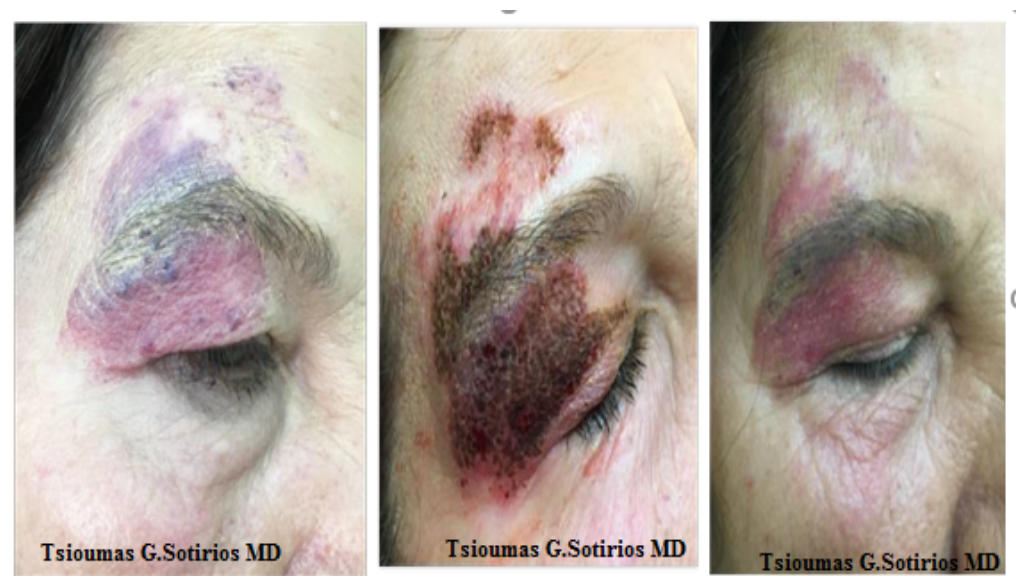

Figure 2. Hemangioma

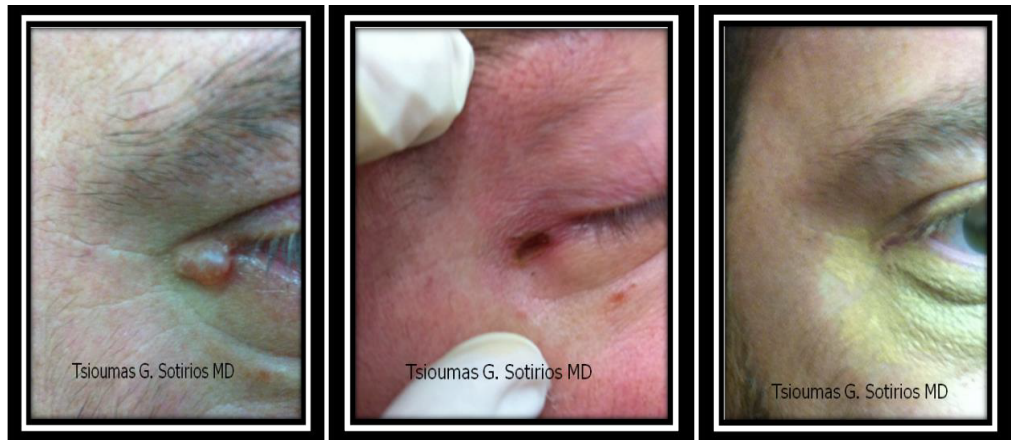

Figure 3. Removal of cysts from the outer cantus with plexr

Syringomi: Spot by spot Method

Papillomi: depending on the size either Spray Method or Spot by Spot Method

Benign Tumors: Spot by spot around the tumor to anesthetize the area and then Spray method to sublimate the tumor

Chalazion: If it's in the gray line follow the spray method, if it is in tarsus use the spray method and the spot by spot method combined (Figure 4.a-f)

Nodules at the gray line: Spray Method

\section{Results}

The obvious result in all cases is the complete sublimation from the first session, without scarring, sutures, and long recovery periods. In cases of ectropion and entropion we may need a second session depending on the degree of ectropion/entropion (2nd or 3rd degree).

Plasma Exeresis method is effective not only because the patient avoids surgery having the same effect with less cost but also for histological reasons. Histological analyzes (Figure 5a-e) before and after show that in area that Plasma Exeresis was applied, by sublimating 


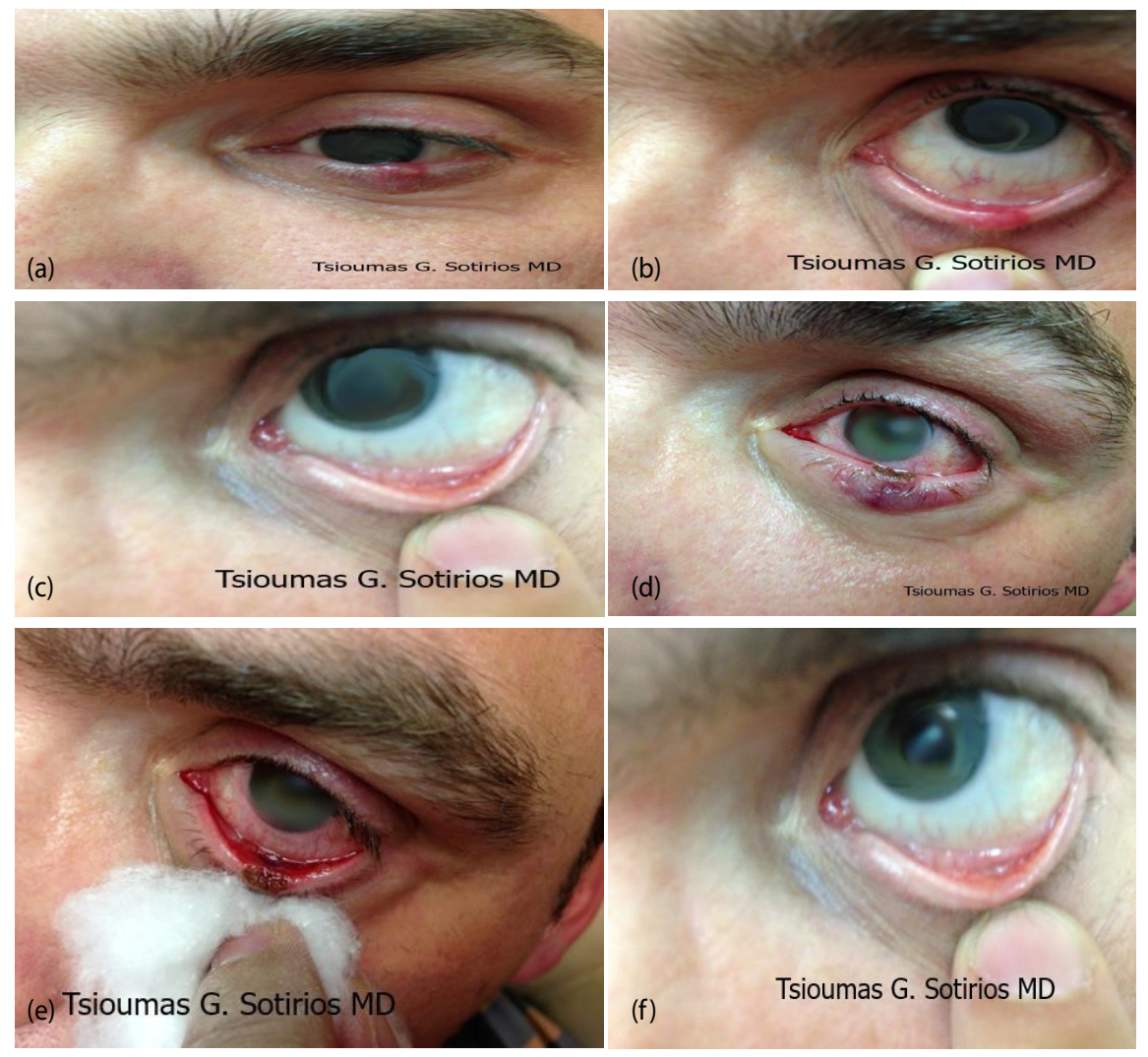

Figure 4. Chalazion removal on the lower eyelid with plexr without eye closure
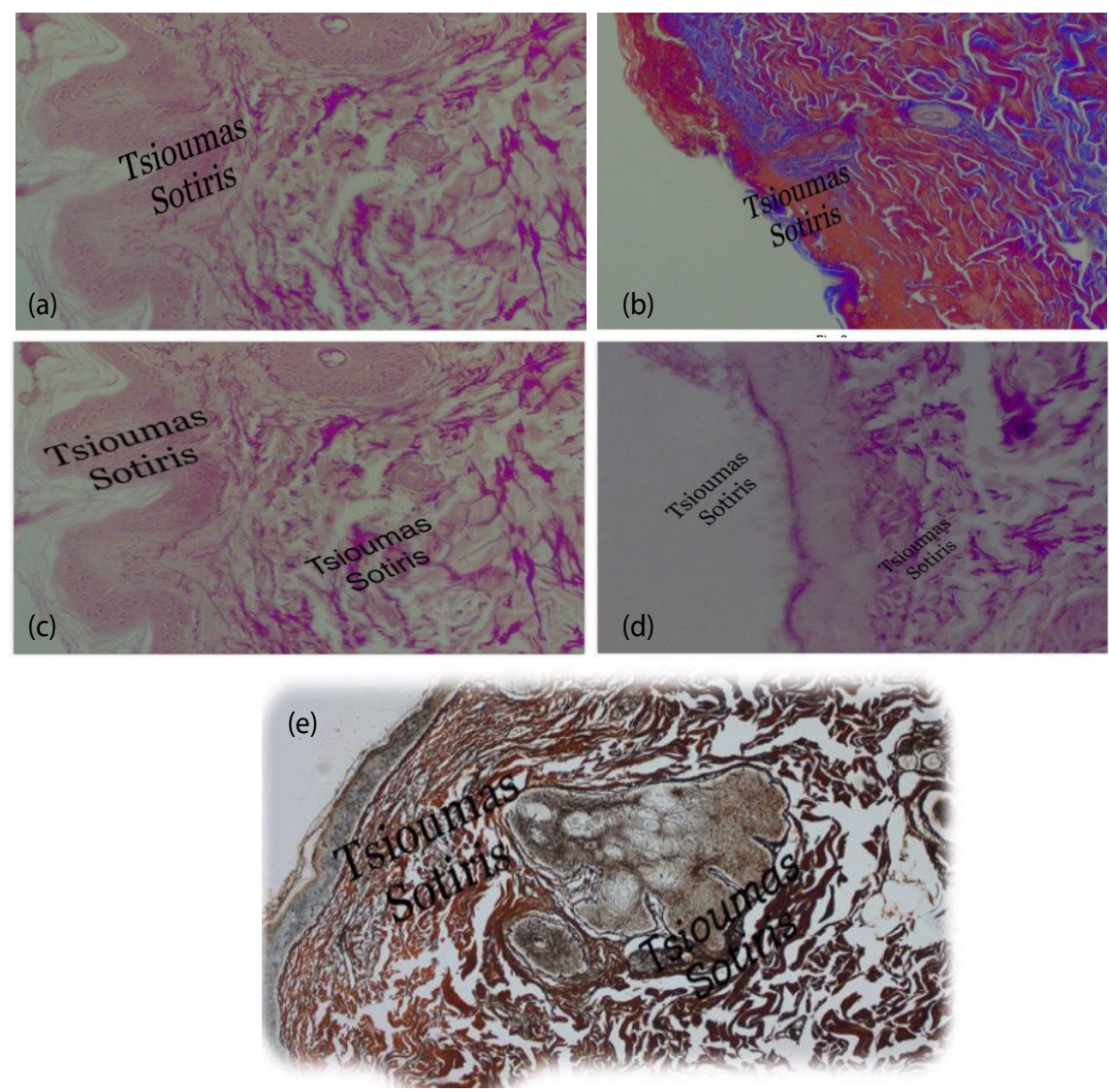

Figure 5. Histological analysis 
the epidermal without going through the basic membrane (Figure 5b), the area not only was reconstructed but collagen type III was created after application (Figure 5c).

\section{Conclusion}

After 347 successful cases, it is a fact that nowdays every doctor is able to provide permanent and effective treatments to the patient using the Plasma Exeresis in his clinic. The patient with low cost and without the fear of surgery solves his problem without blood, stitches and painful procedures. Finally, it is worth to mention that the use of
Plasma Exeresis is not only effective in ophthalmology but in other specialties as well. [1-3]

\section{References}

1. Sotirios G Tsioumas "Manual of Aesthetic Medicine and Non-ablative surgery",2015

2. Tsioumas G Sotiris, Georgiadis Nikolaos, Georgiadou Irini (2014) "Plexr: The Revolution in Blepharoplasty" Pinnacle Medicine \& Medical Sciences 1: 423-424.

3. Georgia Gloustianou, Maria Sifaki, Sotiris G Tsioumas, Vlachodimitropoulos, Antonio Scarano (2016) "Presentation of Old and New Histological Results after Plasma Exeresis(Plexr)Application(Regeneration of The Skin Tissue With Collagen III)". Pinnacle Medicine \& Medical Sciences 3: 983-990.

Copyright: (2017 Sotiris TG. This is an open-access article distributed under the terms of the Creative Commons Attribution License, which permits unrestricted use, distribution, and reproduction in any medium, provided the original author and source are credited. 